\title{
Outcomes of Cardiac Surgery with Very Prolonged Cardiopulmonary Bypass Times
}

\author{
Nicholas Hess ${ }^{1}$, Ibrahim Sultan ${ }^{2}$, Yisi Wang ${ }^{3}$, Floyd Thoma ${ }^{3}$, and Arman Kilic ${ }^{3}$ \\ ${ }^{1}$ University of Pittsburgh Medical Center \\ ${ }^{2}$ University of Pittsburgh \\ ${ }^{3}$ University of Pittsburgh Medical Center Health System
}

March 4, 2021

\begin{abstract}
Background: This study evaluated outcomes of cardiac operations with very prolonged cardiopulmonary bypass (CPB) times. Methods: This was a retrospective study of cardiac surgical procedures with very-long CBP times ([?]300 minutes) performed at a single institution. Primary outcomes of operative ([?]30-day) and one-year mortality were evaluated, and secondary outcomes included use of mechanical support and postoperative complications. A probability analysis was also conducted to identify the correlation between operative mortality and CPB time. Results: 293 patients were included with a Society of Thoracic Surgeons predicted risk of mortality of $2.51 \%$ (IQR 1.32\%-4.70\%), and median CPB time of 340 minutes (IQR 315.0-385.0). Preoperative mechanical support included intra-aortic balloon pump (IABP, $\mathrm{n}=4,1.4 \%$ ), Impella ( $\mathrm{n}=0$ ), or extracorporeal membrane oxygenation $(\mathrm{ECMO}, \mathrm{n}=2,0.7 \%$ ). Intraoperative and operative mortality were $5.1 \%$ and $22.5 \%$, respectively. Intraoperative or postoperative mechanical support were utilized in $90(30.7 \%)$ cases, including IABP (n=65, $22.2 \%)$, Impella $(n=2,0.68 \%)$, or ECMO $(n=23,7.8 \%)$. Postoperative complications included prolonged mechanical ventilation $(\mathrm{n}=121,41.3 \%)$, renal failure $(\mathrm{n}=71,24.2 \%)$, stroke $(\mathrm{n}=20,6.8 \%)$, and reoperation $(\mathrm{n}=95,32.4 \%)$. One-year survival for the overall cohort was $61.4 \%$. Probability analysis identified a CPB time of 446 minutes to be associated with $50 \%$ odds of operative mortality. Conclusions: Cardiac operations requiring very-long CPB times are associated with high rates of operative mortality and morbidity, with a $50 \%$ operative mortality rate predicted for those with a CPB time of 446 minutes or longer. Nonetheless, the majority of patients survive at least 1 year, and these cases should not be considered futile.
\end{abstract}

\section{Introduction}

The development and evolution of cardiopulmonary bypass (CPB) has proven fundamental in the era of cardiac surgery, allowing maintenance of end-organ perfusion during a vast array of surgical procedures ${ }^{1}$. Since its development, improved technologies have aimed at reducing pump-related complications, which include improved oxygenators, pumps, defoaming agent, and heparin-bonded circuits ${ }^{2}$. Despite advancement in this technology over the past century, CPB continues to be a main source of morbidity during cardiac surgical procedures.

Prolonged CPB perfusion times have been associated with various complications such as acute renal injury ${ }^{3-5}$, prolonged mechanical ventilation ${ }^{6}$, and increased intensive care times ${ }^{7}$. Additionally, increased CPB times have also been associated with increased embolic burden in the brain following surgery ${ }^{8}$, and postoperative mortality ${ }^{9-11}$. For these reasons, it is paramount that efficient, expeditious operations be carried out when $\mathrm{CPB}$ is employed to ensure optimal outcomes. However, it is unknown if outcomes following very-long CPB runs should be considered futile. This study evaluated the outcomes of cardiac operations associated with very-long CPB times.

\section{Patients and Methods}




\section{Study Population}

This was a retrospective single institutional analysis that included adult patients (18 years or older) who underwent cardiac surgery at a multi-hospital health system between January 2011 and June 2018. Patients were included if they were placed on CPB during the case and had a perfusion time of 300 minutes or greater. For patients requiring multiple $\mathrm{CPB}$ runs, the cumulative time on $\mathrm{CPB}$ was calculated and utilized for analysis. Patients requiring circulatory arrest were excluded. This study was approved by the Institutional Review Board at the University of Pittsburgh (MOD18120143-003, approved 3/9/2020). Patient consent was waived due to retrospective nature of the study.

\section{Primary and Secondary Outcomes}

The primary outcomes of this study were operative and one-year mortality. Operative mortality was defined as occurring within 30 days of the operation or in-hospital during the index hospitalization following the operation. Secondary outcomes included intraoperative mortality, postoperative complications, and rates of readmission. Use of mechanical support was also evaluated.

\section{Statistical Analysis}

Continuous data are presented as mean \pm standard deviation for normally distributed variables or median [interquartile range (IQR)] for non-normally distributed variables. Categorical data are displayed as number (percentage). Kaplan Meier analysis was used to evaluate one-year survival. A probability analysis was conducted to identify estimated rates of operative mortality based on CPB time over 300 minutes. Statistical analyses were performed using SAS version 9.2 software (SAS Institute, Cary, NC).

\section{Results}

\section{Patient Characteristics and Operative Details}

A total of 293 cardiac surgical patients were included in this study with CPB time [?] 300 minutes. Baseline patient demographics are summarized in Table 1 . Median age and body mass index were 62 years and 28.1 $\mathrm{kg} / \mathrm{m}^{2}$, respectively. Patients were predominately of male sex and white race. The majority of patients were also of New York Heart Association class 1 symptoms (65.2\%) and 32.4\% had a prior history of heart failure. Median Society of Thoracic Surgeons predicted risk of mortality was 2.5\% (IQR 1.3\%-4.7\%). A total of 6 patients $(2.0 \%)$ had preoperative mechanical support (4 intra-aortic balloon pump (IABP), 2 extracorporeal membrane oxygenation (ECMO)).

A breakdown of performed operations are displayed in Table 2 . The most common operation performed in this cohort was aortic root replacement $(34.5 \%)$, followed by double valve procedure $(23.6 \%)$ and combined coronary artery bypass grafting and aortic valve replacement $(9.6 \%)$. Forty-four percent of cases were performed in an elective setting, while $20.5 \%$ were performed as an emergent and/or salvage procedure. A total of $116(39.6 \%)$ cases were redo operations. Median CPB time in this cohort was 340.0 minutes (IQR 315.0 - 385.0 minutes), and median cross clamp time was 254.0 minutes (IQR 204.0 - 298.0 minutes). Median CPB time was longest in the combined CABG and aortic valve replacement group (350.5 minutes, IQR 325.5 - 397.5 minutes). IABP was utilized intraoperatively in $20.1 \%$ of cases, and ECMO was used intraoperatively in $7.2 \%$ of cases (Table 3 ). Postoperative IABP or ECMO were used in $6(2.1 \%)$ and 2 $(0.7 \%)$ cases, respectively. No patients received a ventricular assist device intraoperatively or postoperatively.

\section{Primary and Secondary Outcomes}

Perioperative complications are shown in Table 4 . Fifteen (5.1\%) patients died intraoperatively, and operative mortality was $22.5 \%$. Reoperation and blood transfusions were required in $32.4 \%$ and $66.6 \%$ of patients, respectively. The most common postoperative complication was prolonged mechanical ventilation ( $>24$ hours), followed by atrial fibrillation and renal failure. Median intensive care unit time was 88.9 hours (IQR 42.0 - 205.0 hours).

Probability analysis demonstrated a correlated relationship between CPB time and operative mortality 
(Figure 1) . A CPB time of 353 minutes correlated with an estimated $25 \%$ operative mortality, 446 minutes with $50 \%$ operative mortality, and 539 minutes with $75 \%$ operative mortality.

Median follow up time was 3.27 years (IQR 0.07 -6.42 years). One- and five-year rates of readmission for any cause were $32.8 \%$ and $45.1 \%$, respectively. One-month and one-year survival were $73.7 \%$ and $61.4 \%$, respectively (Figure 2 ).

\section{Discussion}

CPB is arguably one of the greatest developments to allow for the expansion and advancement of the field of cardiac surgery ${ }^{1,12}$. Over the decades, preexisting technologies have improved with the goal of reducing CPBrelated complications. Some of these technologies include centrifugal pumps, improved oxygenators, and heparin-bound circuit tubing ${ }^{2}$. These advancements aim to reduce air emboli and reduce thrombus formation within the CPB circuit, and thus eliminate thromboembolic complications. Despite such advancements, MRI-adjudicated studies have revealed shockingly high rates of new, albeit mostly clinically insignificant, brain lesions following on-pump cardiac surgery ${ }^{13,14}$. Additionally, CPB runs are also linked to elevation of pro-inflammatory cascades, which can result in the development of systemic vasoplegia and end-organ malperfusion ${ }^{15-19}$. Though necessary for the field of cardiac surgery, CPB technology remains an area for improvement.

Because of the known complications and increased mortality risk with prolonged CPB usage ${ }^{9-11}$, it is universally accepted that pump runs be kept to a minimum, and that cardiac procedures be planned accordingly to be conducted in such a way to eliminate unnecessary CPB and cross-clamp times. Nissinen and Madhavan have suggested that the optimal CPB time to be under 180-240 minutes in order to minimize risk of severe complications and/or mortality 7,11 . In the analysis by Nissinen and colleagues, increasing CPB time (per 30 minutes OR 1.47, 95\% CI 1.27-1.71 was associated with increased odds of 30-day mortality, independent of case complexity or patient comorbidities. In their series, only 30 patients had a CPB time longer than 300 minutes, and 30-day mortality was $56.7 \%$ in this cohort. In our cohort of 293 patients, operative mortality was $22.5 \%$, significantly lower than previously described. In an evolving era of cardiac surgery, increasingly complex procedures are being performed, along with complex redo operations, which not surprisingly require lengthier pump runs. Although prior studies have correlated poorer outcomes with increasing CPB times, the current limitations of our existing technology are not well understood.

As stated, increased CPB times have been linked to worse survival, renal, failure, and increased intensive care unit utilization ${ }^{4,7,9,20}$, which likely are associated with increased healthcare costs. There are limited studies that have specifically investigated outcomes of patients in which very-long CPB perfusion times were required. One such study evaluated patients with aortic cross-clamp times exceeding 300 minutes with an average CPB time of 420 minutes, and demonstrated a 30-day mortality of $12.4 \%^{21}$. In patients surviving at least 30 days, the 1-year survival was $92 \%$ in their series, again supporting the notion that longer term survival is favorable if early mortality is avoided in this patient cohort.

As expected, this population was prone to a high degree of complications in our analysis, namely prolonged mechanical ventilation, renal failure, and need for reoperation and blood transfusion. Furthermore, one fifth of patients died within the perioperative window. However, at one year, more than $60 \%$ of patients had survived with roughly one third of patients requiring readmission for any cause. Such findings suggest that although these cases with excessive perfusion times, whether planned or unplanned, are faced with a very high risk of operative mortality and morbidity, mid-term results are acceptable with the majority of patients being alive. Therefore, cardiac operations requiring excessive CPB times should not be considered futile.

The granular causes of very-long CPB times can be multifactorial and were not captured in our data registry. These causes can be categorized as multi-component procedures that require lengthy operative times even if performed efficiently, or cases that should have limited CPB times but where intraoperative complications or issues led to a very prolonged operative course. Nearly $50 \%$ of the cases in our series were multicomponent cases consisting of coronary revascularization combined with a valve procedure, or a multi-valve operation. The most common procedure was an aortic root replacement. By comparison, the mean CPB 
times reported for combined CABG with aortic valve replacement or isolated aortic root replacement are 123203 minutes $^{22,23}$ and $122-237$ minutes $^{24-26}$, respectively. This suggests that even in these typically lengthier cases, the CPB times typically do not exceed 300 minutes. Factors such as having to revise distal anastomoses in CABG, replacing a valve in which a suboptimal repair was performed, or reapplying a cross-clamp to address a paravalvular leak in valve replacement surgery can add time to the operation. Intraoperative complications, although rare, can add substantial operative time, including such events as atrioventricular groove disruption during mitral valve replacement, root disruption during aortic valve surgery, or poor coronary flow after a root replacement requiring coronary revascularization.

The longer $\mathrm{CPB}$ times may also reflect reperfusion time after an unsuccessful attempt at weaning the patient from CPB at the conclusion of the case. Most surgeons, after an initial CPB wean, will empty and reperfuse the heart for a longer period of time if the ventricular function is suboptimal and the patient cannot be weaned successfully and safely from CPB. This can be the result of poor baseline ventricular function, suboptimal myocardial protection, or temporary insults such as air embolism. Protamine reactions can also occur requiring reinstitution of $\mathrm{CPB}$ with added time.

Another important factor in achieving reasonable success in these more complex scenarios is early institution of temporary mechanical circulatory support. This is reflected in the $20 \%$ of patients in whom we utilized an intraoperative IABP. In cases where the patient cannot be weaned from CPB, we typically will attempt weaning with IABP support, and then escalate to ECMO if the patient is still unable to be weaned. In addition, we prefer to utilize IABP support in marginal cases where the patient can be weaned from CPB but is requiring high levels of inotropic support in the setting of depressed ventricular function. The use of mechanical support depends heavily on the surgeon's judgement. However, an increasing body of evidence underscores the importance of these devices in reducing native cardiac stress and distension, improving contractility and myocardial recovery, and increasing end-organ perfusion, all of which contribute to the overall mortality and morbidity of the patient ${ }^{27-31}$.

\section{Limitations}

This study has several limitations. This study is retrospective in design and not randomized. As such, its results may be prone to selection bias which may have influenced the results. The causes of prolonged CPB times were not captured in this data registry but may influence the results. Similarly, details regarding surgical judgement including technical details and use of mechanical support could not be captured. Other factors prone to variability such as intensive care unit care and surgeon experience could potentially confound results as well. Finally, the generalizability of these outcomes to other centers is unknown.

\section{Conclusions}

In this review of 293 consecutive patients undergoing cardiac surgery with CPB pump times [?]300 minutes, we observed reasonable mid-term survival rates, with over $60 \%$ surviving to 1-year, despite high rates of early operative mortality and morbidity. A CPB time of 446 minutes may be useful as a marker of predicting $50 \%$ operative mortality. With appropriate management including use of mechanical circulatory support when needed, cardiac operations requiring very-prolonged CPB times can be salvageable and should not be considered futile.

\section{Acknowledgements}

None

\section{Disclosures}

Ibrahim Sultan, MD receives institutional research support from Atricure and Medtronic and serves as a consultant for Medtronic Vascular. Arman Kilic, MD is on the medical advisory board for Medtronic, Inc. These affiliations do not create direct conflicts with the content of this manuscript.

\section{References}


1. Stoney WS. Evolution of cardiopulmonary bypass. Circulation . 2009;119(21):2844-2853. doi:10.1161/CIRCULATIONAHA.108.830174

2. Sarkar M, Prabhu V. Basics of cardiopulmonary bypass. Indian J Anaesth . 2017;61(9):760-767. doi:10.4103/ija.IJA_379_17

3. Kumar AB, Suneja M, Bayman EO, Weide GD, Tarasi M. Association between postoperative acute kidney injury and duration of cardiopulmonary bypass: A meta-analysis. J Cardiothorac Vasc Anesth . 2012;26(1):64-69. doi:10.1053/j.jvca.2011.07.007

4. Axtell AL, Fiedler AG, Melnitchouk S, et al. Correlation of cardiopulmonary bypass duration with acute renal failure after cardiac surgery. $J$ Thorac Cardiovasc Surg . 2020;159(1):170-178.e2. doi:10.1016/j.jtcvs.2019.01.072

5. Gaudino M, Luciani N, Giungi S, et al. Different profiles of patients who require dialysis after cardiac surgery. Ann Thorac Surg . 2005;79(3):825-829. doi:10.1016/j.athoracsur.2004.08.019

6. Nadeem R, Agarwal S, Jawed S, Yasser A, Altahmody K. Impact of Cardiopulmonary Bypass Time on Postoperative Duration of Mechanical Ventilation in Patients Undergoing Cardiovascular Surgeries: A Systemic Review and Regression of Metadata. Cureus . 2019;11(11). doi:10.7759/cureus.6088

7. Madhavan S, Chan SP, Tan WC, et al. Cardiopulmonary bypass time: Every minute counts. J Cardiovasc Surg (Torino) . 2018;59(2):274-281. doi:10.23736/S0021-9509.17.09864-0

8. Brown WR, Moody DM, Challa VR, Stump DA, Hammon JW. Longer duration of cardiopulmonary bypass is associated with greater numbers of cerebral microemboli. Stroke . 2000;31(3):707-713. doi:10.1161/01.STR.31.3.707

9. Salis S, Mazzanti V V., Merli G, et al. Cardiopulmonary Bypass Duration Is an Independent Predictor of Morbidity and Mortality After Cardiac Surgery. J Cardiothorac Vasc Anesth . 2008;22(6):814-822. doi:10.1053/j.jvca.2008.08.004

10. Salsano A, Giacobbe DR, Sportelli E, et al. Aortic cross-clamp time and cardiopulmonary bypass time: Prognostic implications in patients operated on for infective endocarditis. Interact Cardiovasc Thorac Surg . 2018;27(3):328-335. doi:10.1093/icvts/ivy085

11. Nissinen J, Biancari F, Wistbacka JO, et al. Safe time limits of aortic cross-clamping and cardiopulmonary bypass in adult cardiac surgery. Perfusion . 2009;24(5):297-305. doi:10.1177/0267659109354656

12. Hessel EA. A brief history of cardiopulmonary bypass. Semin Cardiothorac Vasc Anesth . 2014;18(2):87100. doi:10.1177/1089253214530045

13. Wityk RJ, Restrepo L. Cardiac surgery and magnetic resonance imaging of the brain. Arch Neurol . 2002;59(7):1074-1076. doi:10.1001/archneur.59.7.1074

14. Bendszus M, Reents W, Franke D, et al. Brain damage after coronary artery bypass grafting. Arch Neurol . 2002;59(7):1090-1095. doi:10.1001/archneur.59.7.1090

15. Laffey JG, Boylan JF, Cheng DCH. The systemic inflammatory response to cardiac surgery. Anesthesiology . 2002;97(1):215-252. doi:10.1097/00000542-200207000-00030

16. Halter J, Steinberg J, Fink G, et al. Evidence of systemic cytokine release in patients undergoing cardiopulmonary bypass. $J$ Extra Corpor Technol . 2005;37(3):272-277. /pmc/articles/PMC4680784/?report=abstract. Accessed December 7, 2020.

17. Wan S, Leclerc J-L, Vincent J-L. Cytokine Responses to Cardiopulmonary Bypass: Lessons Learned From Cardiac Transplantation .; 1997.

18. Kerbaul F, Guidon C, Lejeune PJ, Mollo M, Mesana T, Gouin F. Hyperprocalcitonemia is related to noninfectious postoperative severe systemic inflammatory response syndrome associated with cardiovascular 
dysfunction after coronary artery bypass graft surgery. J Cardiothorac Vasc Anesth . 2002;16(1):47-53. doi:10.1053/jcan.2002.29672

19. Shaefi S, Mittel A, Klick J, et al. Vasoplegia After Cardiovascular Procedures-Pathophysiology and Targeted Therapy. J Cardiothorac Vasc Anesth . 2018;32(2):1013-1022. doi:10.1053/j.jvca.2017.10.032

20. Salis S, Mazzanti V V., Merli G, et al. Cardiopulmonary Bypass Duration Is an Independent Predictor of Morbidity and Mortality After Cardiac Surgery. J Cardiothorac Vasc Anesth . 2008;22(6):814-822. doi:10.1053/j.jvca.2008.08.004

21. Shultz B, Timek T, Davis AT, et al. Outcomes in patients undergoing complex cardiac repairs with cross clamp times over 300 minutes. J Cardiothorac Surg . 2016;11(1). doi:10.1186/s13019-016-0501-4

22. Sasaki Y, Hirai H, Hosono M, et al. Adding coronary artery bypass grafting to aortic valve replacement increases operative mortality for elderly (70 years and older) patients with aortic stenosis. Gen Thorac Cardiovasc Surg . 2013;61(11):626-631. doi:10.1007/s11748-013-0232-6

23. Kobayashi KJ, Williams JA, Nwakanma L, Gott VL, Baumgartner WA, Conte J V. Aortic Valve Replacement and Concomitant Coronary Artery Bypass: Assessing the Impact of Multiple Grafts. Ann Thorac Surg . 2007;83(3):969-978. doi:10.1016/j.athoracsur.2006.10.027

24. Mccarthy FH, Bavaria JE, Mcdermott KM, et al. At the Root of the Repair Debate: Outcomes After Elective Aortic Root Replacements for Aortic Insufficiency With Aneurysm. Ann Thorac Surg . 2016;102:1199-1205. doi:10.1016/j.athoracsur.2016.03.071

25. Okamura H, Arakawa M, Kimura N, Yuri K, Yamaguchi A. Contemporary outcomes of composite aortic root replacement in elderly patients.Interact Cardiovasc Thorac Surg . 2020;30(3):443-450. doi:10.1093/icvts/ivz267

26. Gaudino M, Lau C, Munjal M, Avgerinos D, Girardi LN. Contemporary outcomes of surgery for aortic root aneurysms: A propensity-matched comparison of valve-sparing and composite valve graft replacement. $J$ Thorac Cardiovasc Surg . 2015;150(5):1120-1129.e1. doi:10.1016/j.jtcvs.2015.07.015

27. Guan Z, Guan X, Gu K, et al. The effect of different types of mechanical circulatory support on mortality of patients after adult cardiac surgery: A systematic review and meta-analysis. Heart Surg Forum . 2020;23(4):E537-E545. doi:10.1532/hsf.2979

28. Kawaguchi O, Pae WE, Daily BB, Pierce WS. Ventriculoarterial coupling with intra-aortic balloon pump in acute ischemic heart failure.J Thorac Cardiovasc Surg . 1999;117(1):164-171. doi:10.1016/S0022$5223(99) 70482-4$

29. Zehetgruber M, Mundigler G, Christ G, et al. Relation of hemodynamic variables to augmentation of left anterior descending coronary flow by intraaortic balloon pulsation in coronary artery disease. Am $J$ Cardiol . 1997;80(7):951-955. doi:10.1016/S0002-9149(97)00553-5

30. Josiassen J, Helgestad OKL, Udesen NLJ, et al. Unloading using Impella CP during profound cardiogenic shock caused by left ventricular failure in a large animal model: impact on the right ventricle.Intensive Care Med Exp . 2020;8(1):41. doi:10.1186/s40635-020-00326-y

31. Rao P, Khalpey Z, Smith R, Burkhoff D, Kociol RD. Venoarterial Extracorporeal Membrane Oxygenation for Cardiogenic Shock and Cardiac Arrest. Circ Hear Fail . 2018;11(9):e004905. doi:10.1161/CIRCHEARTFAILURE.118.004905

Table 1. Preoperative characteristics and demographics of patients undergoing cardiac surgical procedures with [?]300 minutes of cardiopulmonary bypass perfusion times.

\begin{tabular}{ll}
\hline Characteristics & $\mathbf{N}=\mathbf{2 9 3}$ \\
\hline Age (years) & $62.00(50-70)$
\end{tabular}




\begin{tabular}{|c|c|}
\hline Characteristics & $\mathrm{N}=293$ \\
\hline Female & $96(32.76 \%)$ \\
\hline \multicolumn{2}{|l|}{ Race } \\
\hline White & $263(89.76 \%)$ \\
\hline Black & $24(8.19 \%)$ \\
\hline Other & $6(2.05 \%)$ \\
\hline Body mass index $\left(\mathrm{kg} / \mathrm{m}^{2}\right)$ & $28.14(24.69-32.30)$ \\
\hline Body surface area $\left(\mathrm{m}^{2}\right)$ & $2.03(0.28)$ \\
\hline Diabetes mellitus & $58(19.80 \%)$ \\
\hline Dialysis dependency & $11(3.75 \%)$ \\
\hline Chronic obstructive pulmonary disease & $75(25.60 \%)$ \\
\hline Hypertension & $218(74.40 \%)$ \\
\hline Immunosuppression & $24(8.19 \%)$ \\
\hline Cerebrovascular disease & $71(24.23 \%)$ \\
\hline Peripheral vascular disease & $96(32.76 \%)$ \\
\hline Previous myocardial infarction & $70(23.89 \%)$ \\
\hline \multicolumn{2}{|l|}{ NYHA class symptoms } \\
\hline 1 & $191(65.19 \%)$ \\
\hline 2 & $13(4.44 \%)$ \\
\hline 3 & $45(15.36 \%)$ \\
\hline 4 & $44(15.02 \%)$ \\
\hline History of $\mathrm{CHF}$ & $92(31.40 \%)$ \\
\hline Cardiac arrhythmia & $114(38.91 \%)$ \\
\hline Serum creatinine $(\mathrm{mg} / \mathrm{dL})$ & $1.00(0.80-1.20)$ \\
\hline Total bilirubin (mg/dL) & $0.80(0.50-1.10)$ \\
\hline Total albumin (g/dL) & $? i ?$ \\
\hline 3.20 & $62(21.16 \%)$ \\
\hline $3.20-3.70$ & $69(23.55 \%)$ \\
\hline $3.70-4.00$ & $41(13.99 \%)$ \\
\hline$>4.00$ & $57(19.45 \%)$ \\
\hline Not measured & $64(21.84 \%)$ \\
\hline Left ventricular ejection fraction (\%) & $55.00(50.00-60.00)$ \\
\hline STS predicted risk of mortality (\%) & $2.51(1.32-4.70)$ \\
\hline
\end{tabular}

Data is presented as number (\%), median (interquartile range), or mean ( \pm standard deviation)

CHF, congestive heart failure

STS, Society of Thoracic Surgeons

Table 2. Description and operative characteristics of cardiac surgical cases requiring [?]300 minutes of cardiopulmonary bypass perfusion time.

\begin{tabular}{lll}
\hline Operation Performed & N (\%) & Median Perfusion Time, minutes (IQR) \\
\hline Aortic Root & $101(34.47 \%)$ & $344.00(315.00,379.00)$ \\
CABG + AVR & $28(9.56 \%)$ & $350.50(325.50,397.50)$ \\
CABG + MVr/MVR & $14(4.78 \%)$ & $344.50(315.00,383.00)$ \\
Double valve & $69(23.55 \%)$ & $347.00(318.00,389.00)$ \\
Isolated AVR & $14(4.78 \%)$ & $346.00(330.00,399.00)$ \\
Isolated CABG & $9(3.07 \%)$ & $316.00(307.00,323.00)$ \\
Isolated MVr/MVR & $17(5.80 \%)$ & $323.00(312.00,345.00)$
\end{tabular}




\begin{tabular}{lll}
\hline Operation Performed & N (\%) & Median Perfusion Time, minutes (IQR) \\
\hline Triple Valve & $21(7.17 \%)$ & $335.00(316.00,421.00)$ \\
$\begin{array}{l}\text { Other } \\
\text { Operative Details }\end{array}$ & $20(6.83 \%)$ & $327.00(308.00,424.00)$ \\
History of prior cardiac surgery & $116(39.56 \%)$ & $116(39.56 \%)$ \\
Operative status & & \\
Elective & $129(44.03 \%)$ & $129(44.03 \%)$ \\
Urgent & $104(35.49 \%)$ & $104(35.49 \%)$ \\
Emergent or salvage & $60(20.48 \%)$ & $60(20.48 \%)$ \\
Perfusion time (minutes) & $340.0(315.0-385.0)$ & $340.0(315.0-385.0)$ \\
Cross clamp time (minutes) & $254.0(204.0-298.0)$ & $254.0(204.0-298.0)$ \\
\hline
\end{tabular}

AVR, aortic valve replacement

CABG, coronary artery bypass graft

IQR, interquartile range

$\mathrm{MVr}$, mitral valve repair

MVR, mitral valve replacement

Table 3. Preoperative, intraoperative, and postoperative usage of mechanical circulatory support in patients undergoing cardiac surgery with [?]300 minutes of cardiopulmonary bypass perfusion time.

\begin{tabular}{ll}
\hline Mechanical Support & N (\%) \\
\hline Intra-aortic balloon pump placement & \\
None & $224(76.45 \%)$ \\
Preoperative & $4(1.37 \%)$ \\
Intraoperative & $59(20.14 \%)$ \\
Postoperative & $6(2.05 \%)$ \\
Impella placement & \\
None & $291(99.32 \%)$ \\
Preoperative & $0(0.00 \%)$ \\
Intraoperative & $2(0.68 \%)$ \\
Postoperative & $0(0.00 \%)$ \\
ECMO placement & \\
None & $268(91.47 \%)$ \\
Preoperative & $2(0.68 \%)$ \\
Intraoperative & $21(7.17 \%)$ \\
Postoperative & $2(0.68 \%)$ \\
Ventricular assist device placement & $0(0.00 \%)$ \\
\hline
\end{tabular}

ECMO, extracorporeal membrane oxygenation

Table 4. Postoperative complications and outcomes of patients undergoing cardiac surgery with [?]300 minutes of cardiopulmonary bypass perfusion time.

\begin{tabular}{ll}
\hline & $\mathbf{N}(\boldsymbol{\%})$ \\
\hline Operative mortality & $66(22.53 \%)$ \\
Intraoperative mortality & $15(5.12 \%)$
\end{tabular}




\begin{tabular}{ll}
\hline & N (\%) \\
\hline Reoperation & $95(32.42 \%)$ \\
Blood transfusion & $195(66.55 \%)$ \\
Prolonged mechanical ventilation $(>24$ hours) & $121(41.30 \%)$ \\
Pneumonia & $44(15.02 \%)$ \\
Renal failure & $71(24.23 \%)$ \\
Stroke & $20(6.83)$ \\
Sepsis & $18(6.14 \%)$ \\
Superficial wound infection & $9(3.07 \%)$ \\
Deep sternal wound infection & $3(1.02 \%)$ \\
New atrial fibrillation & $78(26.62 \%)$ \\
Median ICU time (hours) & $88.85(42.00-205.00)$ \\
\hline
\end{tabular}

ICU, intensive care unit

Figure Legend

Figure 1. Predicted odds of mortality plotted over increasing cardiopulmonary bypass time. Blue band represents $95 \%$ confidence interval

Figure 2. Kaplan Meier estimate of one-year survival in patients undergoing cardiac surgery with [?]300 minutes of cardiopulmonary bypass time

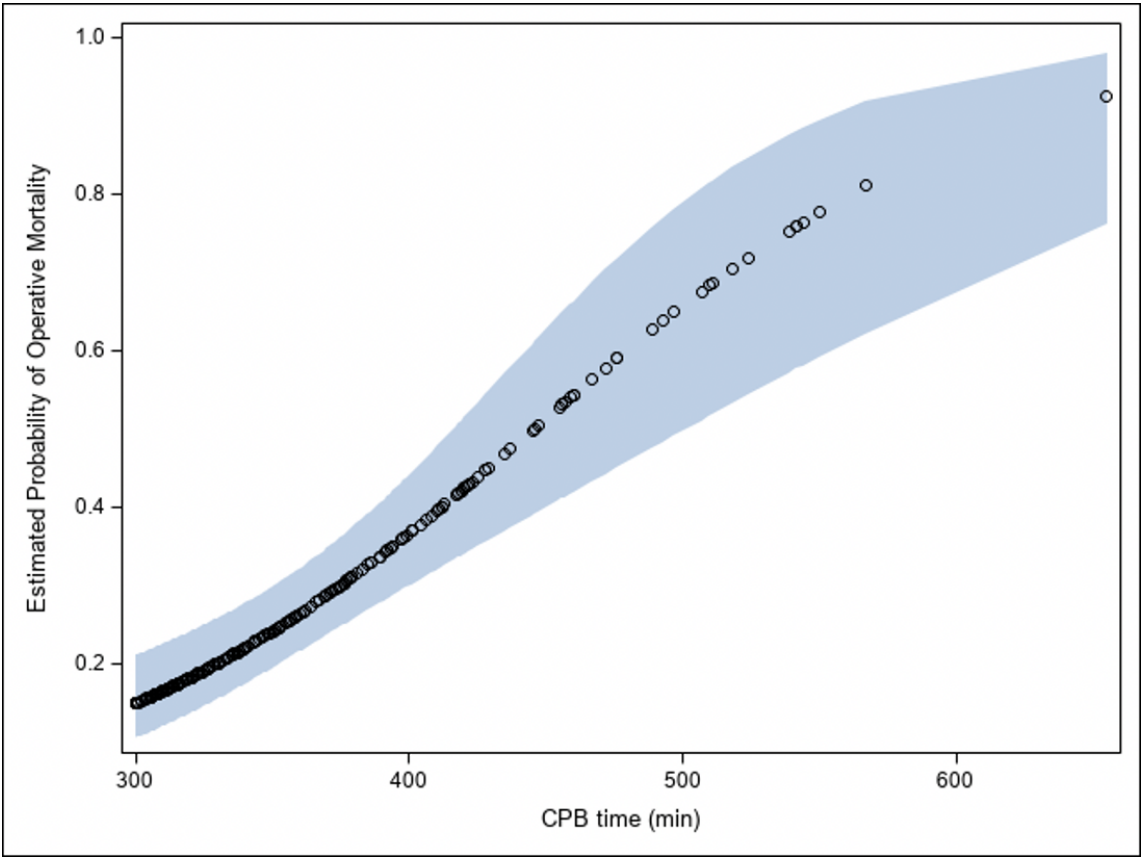




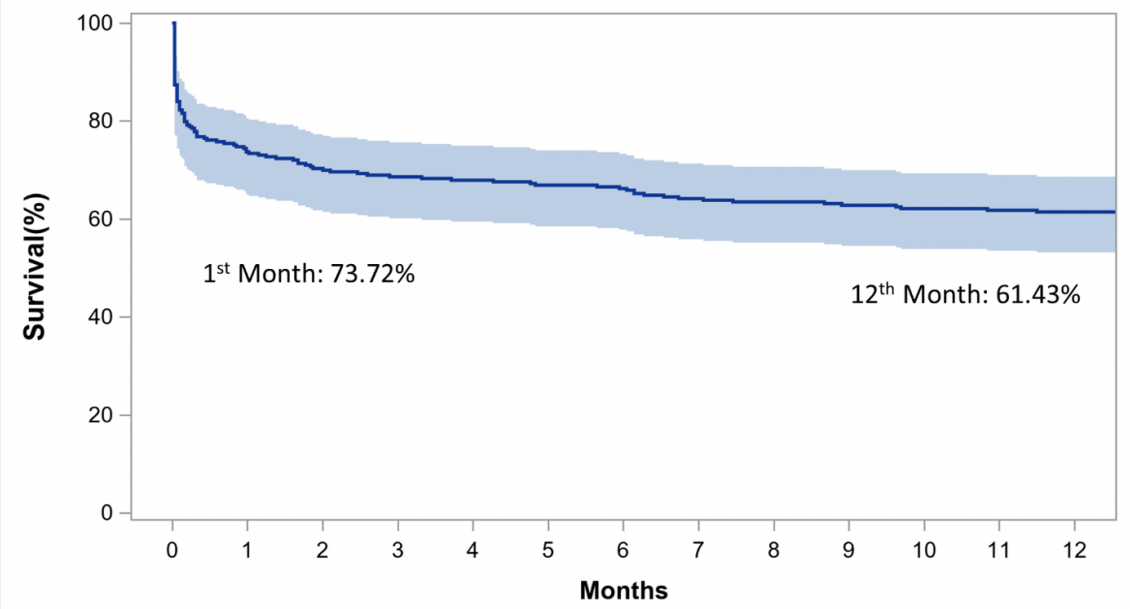

$\square \% 95$ Confidence Interval Band

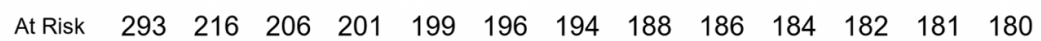

